\title{
Incidence of breast cancer and estimates of overdiagnosis after the initiation of a population-based mammography screening program
}

\author{
Andrew Coldman PhD, Norm Phillips MSc
}

\author{
Competing interests: \\ Andrew Coldman was \\ previously associated with \\ the Screening \\ Mammography Program of \\ $\mathrm{BC}$ in an administrative \\ capacity; he is currently \\ employed by the BC Cancer \\ Agency, which manages \\ this program. Norm Phillips \\ is currently employed by \\ the BC Cancer Agency, \\ which manages the \\ Screening Mammography \\ Program of BC.
}

This article has been peer reviewed.

Correspondence to: acoldman@bccancer.bc.ca

CMAJ 2013. DOI:10.1503 /cmaj.121791

\section{ABSTRACT \\ Background: There has been growing interest in the overdiagnosis of breast cancer as a result of mammography screening. We report incidence rates in British Columbia before and after the initiation of population screening and provide estimates of overdiagnosis.}

Methods: We obtained the numbers of breast cancer diagnoses from the BC Cancer Registry and screening histories from the Screening Mammography Program of $\mathrm{BC}$ for women aged 30-89 years between 1970 and 2009. We calculated age-specific rates of invasive breast cancer and ductal carcinoma in situ. We compared these rates by age, calendar period and screening participation. We obtained 2 estimates of overdiagnosis from cumulative cancer rates among women between the ages of 40 and 89 years: the first estimate compared participants with nonparticipants; the second estimate compared observed and predicted population rates.

Results: We calculated participation-based estimates of overdiagnosis to be $5.4 \%$ for invasive disease alone and $17.3 \%$ when ductal carcinoma in situ was included. The corresponding population-based estimates were $-0.7 \%$ and $6.7 \%$. Participants had higher rates of invasive cancer and ductal carcinoma in situ than nonparticipants but lower rates after screening stopped. Population incidence rates for invasive cancer increased after 1980; by 2009, they had returned to levels similar to those of the 1970s among women under 60 years of age but remained elevated among women $60-79$ years old. Rates of ductal carcinoma in situ increased in all age groups.

Interpretation: The extent of overdiagnosis of invasive cancer in our study population was modest and primarily occurred among women over the age of 60 years. However, overdiagnosis of ductal carcinoma in situ was elevated for all age groups. The estimation of overdiagnosis from observational data is complex and subject to many influences. The use of mammography screening in older women has an increased risk of overdiagnosis, which should be considered in screening decisions.
$\mathrm{T}$ here is growing interest in the overdiagnosis of breast cancer resulting from mammography screening. ${ }^{1,2}$ It has been suggested that incidence rates after the introduction of mammography screening are higher than would be expected from the early detection of clinically significant disease alone. The clinical importance of ductal carcinoma in situ has long been unclear, and recent attention has also focused on the potential overdiagnosis of invasive cancer. ${ }^{1,3,4}$ Furthermore, more frequent screening, wider age ranges and higher false-positive rates in much of North America may result in increased overdiagnosis ${ }^{5}$ in comparison with Europe, the source of most reports.

The Screening Mammography Program of BC provides bilateral 2-view mammography screening to female residents of British Columbia. The program began in 1988 at a single location and progressively expanded by adding centres in larger communities and extending mobile services; by 2000, geographic coverage was effectively $100 \%$. Women aged $40-79$ years are eligible to self-refer. Since 1997, women aged 40-49 years are reminded to return annually, and those over 50 years of age are reminded to return biennially. Women outside of this age-range can undergo screening with a physician's referral. Rates of abnormal results on screening mammography in British Columbia are comparable to the community rates seen in the United States. ${ }^{6}$

Our primary objective for this study was to estimate the extent of overdiagnosis of breast cancer due to screening. To do this, we examined cancer rates in the following subpopulations: all women 1970-1979, and women in 2005-2009 classified by active and nonactive participation in screening. From 1970 to 1979 , screening was infrequent and no organized program existed. In the period 2005-2009, screening was well established and the use of hormone replacement ther- 
apy for menopause had declined. ${ }^{7}$ Our secondary objective was to determine the changes in population incidence of breast cancer, both invasive and ductal carcinoma in situ, following the implementation of a population-based screening program. Thus, we report an analysis of population age-specific incidence rates in British Columbia over a 40-year period (1970-2009).

\section{Methods}

\section{Study population and data sources}

We obtained data on invasive breast cancer and ductal carcinoma in situ diagnosed in female residents of British Columbia between 1970 and 2009 from the BC Cancer Registry. We obtained estimates of population size for the same period from BCStats. We extracted data on identifiers and mammography screens performed between 1988 (the screening program's inception) and 2009 from the Screening Mammography Program of $\mathrm{BC}$ database, which is a complete longitudinal record of all screens performed as part of the program. We used health plan identifiers, names and birthdates to link screening data to the data from the Vital Statistics Agency of British Columbia record of deaths and the $\mathrm{BC}$ Cancer Registry to identify deaths and cases of breast cancer.

\section{Outcome measures}

We calculated cancer detection rates at first screen (either invasive or ductal carcinoma in situ) as the number of cases detected divided by the number of women who underwent screening. For all other analyses, we calculated incidence rates of breast cancer by dividing the number of cases by the total time-at-risk. Where applicable, rates were standardized to the 1991 Canadian population.

We used 2 methods to estimate overdiagnosis. Each method relied on estimated cumulative rates of breast cancer among women between the ages of 40 and 89 years for the period 2005-2009. For the first method (the participation method), overdiagnosis was estimated using the difference between the cumulative rates of breast cancer among women undergoing active screening and women who never underwent screening or who discontinued screening (nonactive screening). For the second method (the population method), overdiagnosis was estimated using the difference between observed and predicted population cumulative rates in 2005-2009. The predicted rates were based on trends in 1970-1979.

For the participation method, each woman's time-at-risk in 2005-2009 was dynamically allocated by attained age to 2 mutually exclusive states corresponding to periods of active and nonactive screening (Figure 1). A woman was considered to be in an active screening state after undergoing a screen until either 60 months $(5 \mathrm{yr})$ had elapsed without screening or she died. Following a diagnosis of breast cancer or reaching 80 years of age, a woman remained in her current state (i.e., active or nonactive).

We chose a period of 60 months to represent an interval in which rates would likely be influenced by previous screening based on estimates of sojourn times. ${ }^{8}$ Women who were in the active screening group stayed there after 80 years of age to identify any compensatory drop in rates (due to early detection) when screening ceased. ${ }^{9}$

We calculated total age-specific activescreening times-at-risk by summing contributions from individual women. We determined total agespecific nonactive-screening times-at-risk by subtracting the corresponding total for active screening from the total times-at-risk obtained from published mid-year population totals. Women who never underwent screening thus contribute to the nonactive screening times-at-risk. Breast cancer diagnoses, other than those determined by the results of a first screen, were allocated to age-specific active and nonactive screening states according to the age and state of the woman at diagnosis.

We calculated age-specific detection rates at first screen for women first undergoing mammography in 2005-2009. We calculated age-specific incidence rates for women in both the active and nonactive screening states. We calculated the cumulative rates of cancer between the ages of 40 and 89 years by summing the relevant agespecific incidence rates. To calculate the cumulative rate while in active screening, we added the first-screen detection rate at age 40 to reflect the impact of the first screen on cumulative risk.

For the population method, we obtained pre-

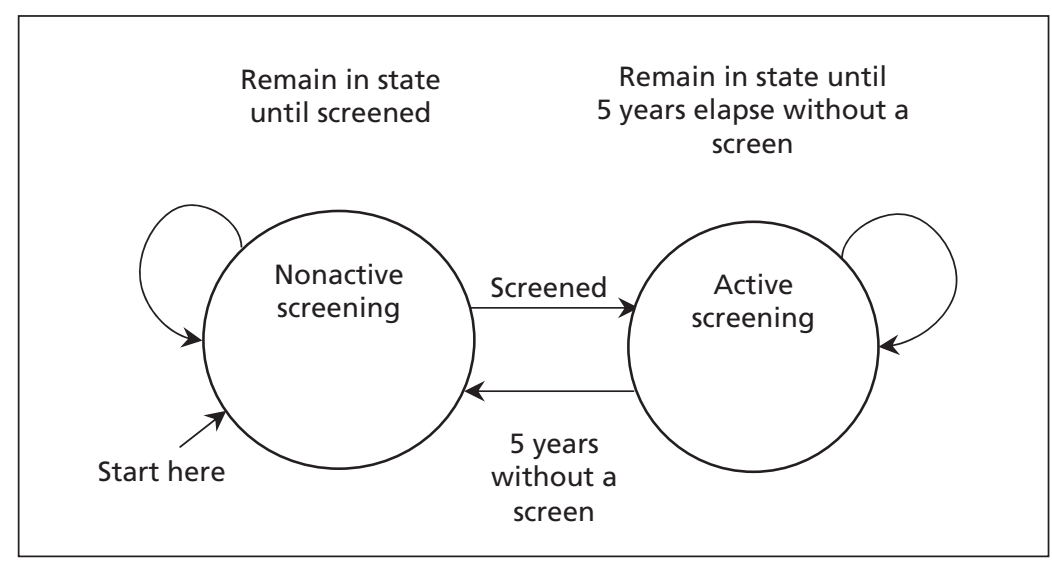

Figure 1: Schematic of the allocation of time-at-risk to active and nonactive screening states. A woman was considered to be in active screening after undergoing screening until either 5 years had elapsed without a mammography screen or she died. A woman remained in her current screening state after a diagnosis of breast cancer or reaching 80 years of age. 
dicted age-specific rates of invasive cancer in 2005-2009 by projecting linear calendar trends over 1970-1979 within 10-year age groups. The projected cumulative rate of ductal carcinoma in situ for 2005-2009 in the absence of screening was taken to be the observed rate in the nonactive screening group in 2005-2009.

\section{Statistical analysis}

We calculated age-specific incidence rate ratios and $95 \%$ confidence intervals (CIs) by comparing 2005-2009 to 1970-1979 using Poisson regression. We estimated linear calendar trend rate ratios of age-specific invasive cancer during 1970-1979 using this method, which we then used to predict age-specific incidence rates in 2005-2009 in the absence of screening. Rate ratio CIs were based on the Wald statistic for a Poisson distribution. We calculated the variances of the cumulative rates as the sums of the variances of the age-specific rates obtained from the Poisson distribution.

\section{Results}

The number of screens performed by the Screening Mammography Program of BC increased substantially in the 1990s as the program expanded to provide service throughout the province. Few women under 40 years of age or older than 80 years of age underwent screening during this period (Table 1). Of the 1387197 screens performed in 2005-2009 among women aged 40-89 years, 197928 (14.3\%) were first screens. The modal ages of women undergoing their first screen were 40-41 years (23.3\%), corresponding to initial eligibility at the age of 40 , and $50-51$ years (8.5\%), corresponding to targeted invitation letters sent to women aged 50 . The number of in situ cancers increased rapidly as the program expanded, although the start of the increase predated widespread organized screening and was likely related to screening outside of the program. Numbers of invasive breast cancers increased over the study period, but this seems to

Table 1: Distribution of cases of breast cancer and ductal carcinoma in situ, mammography screenings and population years-at-risk, by age group and period

\begin{tabular}{|c|c|c|c|c|c|}
\hline \multirow[b]{2}{*}{ Period } & \multirow[b]{2}{*}{ Age group, yr } & \multirow[b]{2}{*}{ Years-at-risk } & \multirow[b]{2}{*}{$\begin{array}{l}\text { Mammography } \\
\text { screenings, no. }\end{array}$} & \multicolumn{2}{|c|}{ No. of cases } \\
\hline & & & & $\begin{array}{l}\text { Invasive breast } \\
\text { cancer }\end{array}$ & $\begin{array}{c}\text { Ductal } \\
\text { carcinoma in } \\
\text { situ }\end{array}$ \\
\hline \multirow[t]{6}{*}{ 1970-1979 } & $30-39$ & 1537498 & 0 & 566 & 22 \\
\hline & $40-49$ & 1310140 & 0 & 1821 & 101 \\
\hline & $50-59$ & 1249583 & 0 & 2703 & 106 \\
\hline & $60-69$ & 943503 & 0 & 2483 & 71 \\
\hline & 70-79 & 558996 & 0 & 1641 & 54 \\
\hline & $\geq 80$ & 298675 & 0 & 966 & 30 \\
\hline \multirow[t]{6}{*}{ 1980-1989 } & $30-39$ & 2422444 & 56 & 914 & 40 \\
\hline & $40-49$ & 1668976 & 5538 & 2267 & 173 \\
\hline & $50-59$ & 1415857 & 4297 & 2934 & 198 \\
\hline & $60-69$ & 1342795 & 3066 & 4065 & 199 \\
\hline & $70-79$ & 868461 & 796 & 3140 & 159 \\
\hline & $\geq 80$ & 428466 & 74 & 1704 & 42 \\
\hline \multirow[t]{6}{*}{ 1990-1999 } & $30-39$ & 3192387 & 4180 & 1136 & 106 \\
\hline & $40-49$ & 2782701 & 441338 & 3878 & 638 \\
\hline & $50-59$ & 1842518 & 377433 & 4392 & 691 \\
\hline & $60-69$ & 1521985 & 286919 & 5100 & 657 \\
\hline & 70-79 & 1228348 & 163148 & 5100 & 513 \\
\hline & $\geq 80$ & 659604 & 15653 & 2700 & 133 \\
\hline \multirow[t]{6}{*}{ 2000-2009 } & $30-39$ & 3042072 & 3217 & 1132 & 103 \\
\hline & $40-49$ & 3472765 & 871237 & 4790 & 1005 \\
\hline & $50-59$ & 2908523 & 803709 & 6377 & 1279 \\
\hline & $60-69$ & 1893532 & 532565 & 5950 & 1059 \\
\hline & $70-79$ & 1377651 & 300283 & 5055 & 748 \\
\hline & $\geq 80$ & 981835 & 12693 & 3375 & 159 \\
\hline
\end{tabular}


have been primarily driven by population aging and growth, as suggested by the changes in person years-at-risk during each period (Table 1).

Age-standardized rates of invasive cancer in women over 60 years of age tended to peak around 1990, whereas the rates in women less than 60 years of age showed no consistent pattern (Figure 2). Linear calendar trends in age-specific rates during the period 1970-1979 were not significant in any age group (Table 2). Rates of invasive breast cancer did not decline significantly in any age group and significantly increased only for women aged 60-79 years (Table 2). After 1980, rates of ductal carcinoma in situ increased in all age groups but appeared to level off after 2000 (Figure 3). Using the rates from 1970-1979 as the reference groups, the rate ratio for ductal carcinoma in situ for the period 2005-2009 was significantly increased for all women under 80 years of age and showed the highest increases in age groups eligible for screening (Table 2).

The rate of invasive cancer detected at a first screen generally increased with age, but the rate of ductal carcinoma in situ showed no clear relation to age (Table 3 ). Dividing the detection rate for invasive cancer at first screen (Table 3 ) by the incidence rate in the active screening group (Table 3 ) provides an indication of the lead time associated with screening. This ratio was less than 2 in women aged 40-44 years but exceeded 4 in women beyond age 75, suggesting lead time increases with age, and shows the potential influence of first screens on overall rates in the population. During nonactive screening periods in 2005 2009 (Table 3), rates of invasive disease were lower in women under age 60 and higher in women over age 70 than population rates in 1970 1979 ; in contrast, rates of ductal carcinoma in situ were higher in all age groups in 2005-2009.

Rates of invasive disease in 2005-2009 were higher in the active screening group than in the nonactive screening group for all ages, except for woman more than 80 years old. After the age of 80 years, there was a compensatory drop in rates (Table 3). The estimated cumulative rate of invasive disease for a woman starting screening at age 40 and continuing to age 89 was 137 per 1000 , compared with 130 per 1000 among women in the nonactive screening state. Thus, the participation estimate of overdiagnosis is $5.4 \%$ (7/130) $(17.3 \%$ [24/139] when ductal carcinoma is included).

Applying age-specific linear calendar trend rate ratios (Table 2) to the corresponding agespecific rates of invasive cancer for 1970-1979

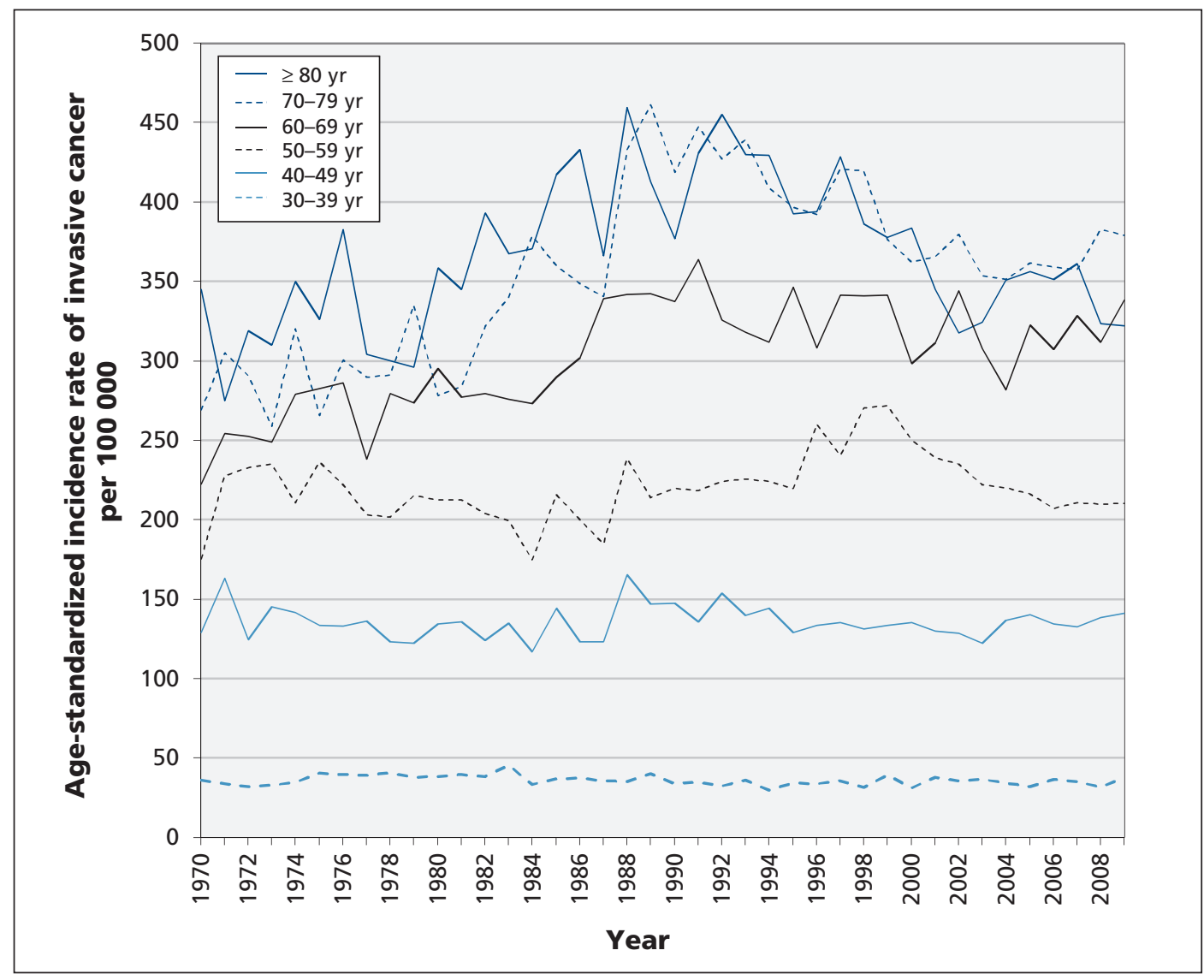

Figure 2: Age-standardized incidence rates of invasive breast cancer among women in British Columbia by age and year of diagnosis. 
(Table 3) resulted in projected age-specific rates for 2005-2009, which were summed to give projected cumulative rates for ages $40-89$ years. The projected cumulative rate is 141 per 100 (150 per 1000 when ductal carcinoma in situ is included). Thus, the population estimate of overdiagnosis of invasive breast cancer is $-0.7 \%(-1 / 141)(6.7 \%$ [10/150] with inclusion of ductal carcinoma in situ). However, CIs for the projected cumulative rate of invasive breast cancer are wide: the $95 \%$ CI for the population estimate of overdiagnosis is $-21 \%$ to $30 \%$.

\section{Interpretation}

Among women undergoing screening for breast cancer through the Screening Mammography Program of $\mathrm{BC}$, the rates of invasive breast cancer in 2005-2009 were significantly elevated for women

\begin{tabular}{|c|c|c|c|c|c|c|}
\hline \multirow[b]{3}{*}{ Age, yr } & \multirow{2}{*}{\multicolumn{2}{|c|}{$\begin{array}{l}\text { Linear calendar trends } \\
1970-1979\end{array}$}} & \multicolumn{4}{|c|}{$\begin{array}{l}\text { Population incidence for } 2005-2009 \text { v. } \\
\text { 1970-1979 (reference) }\end{array}$} \\
\hline & & & \multicolumn{2}{|c|}{ Invasive breast cancer } & \multicolumn{2}{|c|}{ Ductal carcinoma in situ } \\
\hline & RR per year & $95 \% \mathrm{Cl}$ & $\mathrm{RR}$ & $95 \% \mathrm{Cl}$ & $\mathrm{RR}$ & $95 \% \mathrm{Cl}$ \\
\hline $30-39$ & 1.02 & $0.99-1.05$ & 0.92 & $0.82-1.04$ & 2.02 & $1.27-3.23$ \\
\hline $40-49$ & 0.99 & $0.97-1.00$ & 1.01 & $0.95-1.07$ & 4.10 & $3.31-5.08$ \\
\hline $50-59$ & 1.00 & $0.99-1.01$ & 0.97 & $0.92-1.02$ & 4.64 & $3.80-5.67$ \\
\hline $60-69$ & 1.01 & $1.00-1.03$ & 1.21 & $1.15-1.28$ & 7.49 & $5.87-9.57$ \\
\hline 70-79 & 1.01 & $0.99-1.03$ & 1.25 & $1.18-1.33$ & 6.43 & $4.79-8.62$ \\
\hline$\geq 80$ & 1.00 & $0.98-1.02$ & 1.06 & $0.98-1.15$ & 1.40 & $0.95-2.05$ \\
\hline
\end{tabular}

Note: $\mathrm{Cl}=$ confidence interval, $\mathrm{RR}=$ rate ratio

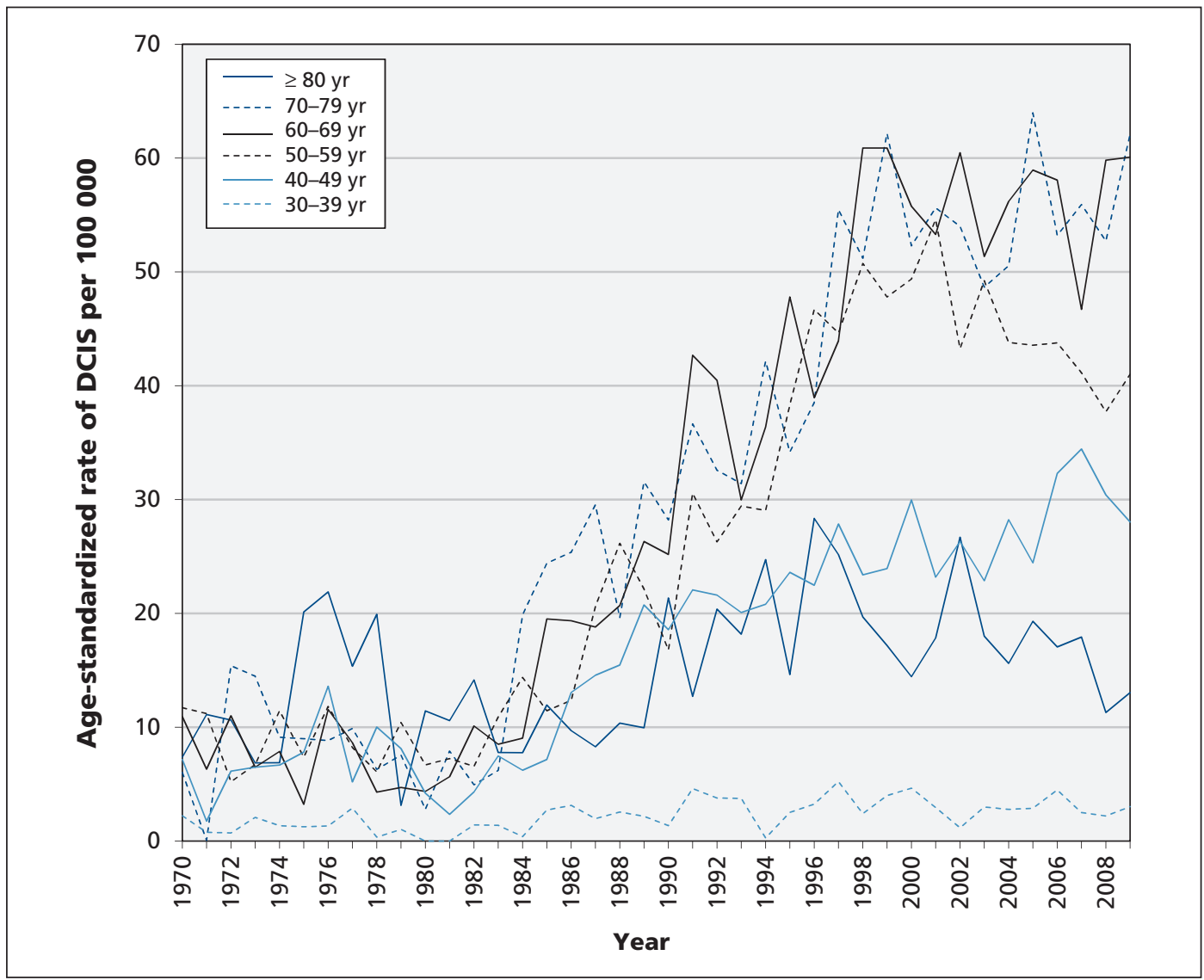

Figure 3: Age-standardized incidence rates of ductal carcinoma in situ (DCIS) among women in British Columbia by age and year of diagnosis. 
between the ages of 60 and 79 years, compared with 1970-1979. Although participation in screening was common among women aged $40-59$ years, no sustained increase was seen in this age group. These results suggest that the effect of screening on overdiagnosis may be age dependent.

Rates of invasive disease for active and nonactive screening states were higher in 2005-2009 than in the whole population in 1970-1979, suggesting that the rate of breast cancer had increased because of changes in population risk. Observed trends in invasive cancer incidence in the 19701979 prescreening period were modest. Increasing lead time with age, as shown by an increasing ratio between cancer detected at first screen and the incidence rate for active screening, suggests that the time required for a compensatory drop will increase with age. Conversely, the low ratios for women under 50 years of age suggests short lead times and low potential for overdiagnosis, as was seen in a study from Sweden. ${ }^{10}$

Long-term follow-up of clinical trials of mammography screening provide the most rigorous method to measure the extent of overdiagnosis. ${ }^{9}$ Moss and colleagues ${ }^{11}$ reviewed findings from clinical trials and included results on both invasive and in situ disease; no increase was seen in trials where intervention and control groups underwent screening at trial conclusion, whereas increases ranging from $4 \%$ to $31 \%$ were found in trials where screening did not occur at trial conclusion. These results suggest that the duration of screening may not be an important influence on rates of overdiagnosis.

Several authors have reported on changes in population incidence rates of breast cancer after the institution of screening for the disease. . $^{1,2,4,12}$ Jørgenson and Gotzsche ${ }^{3}$ reviewed trend-based estimates of overdiagnosis, including one from Canada, and derived an overdiagnosis estimate of $52 \%$ for invasive cancer alone. The studies included in their review showed large increases in the incidence of invasive breast cancer following the introduction of screening, an effect we did not see in British Columbia.

A review of European studies summarized estimates of overdiagnosis, ${ }^{13}$ and companion articles provided commentary on methodology issues $^{14-16}$ in the estimation of overdiagnosis. The European review found that, for estimates considered to be adequately adjusted, overdiagnosis ranged from $1 \%-10 \%$. These are in agreement with the results we report, because we controlled for biases that have been previously identified..$^{16}$

A study from the United States ${ }^{17}$ using data from the Surveillance, Epidemiology and End Results (SEER) Program compared population rates of breast cancer over 1976-2008 among women over 40 years of age and found a large increase, with an

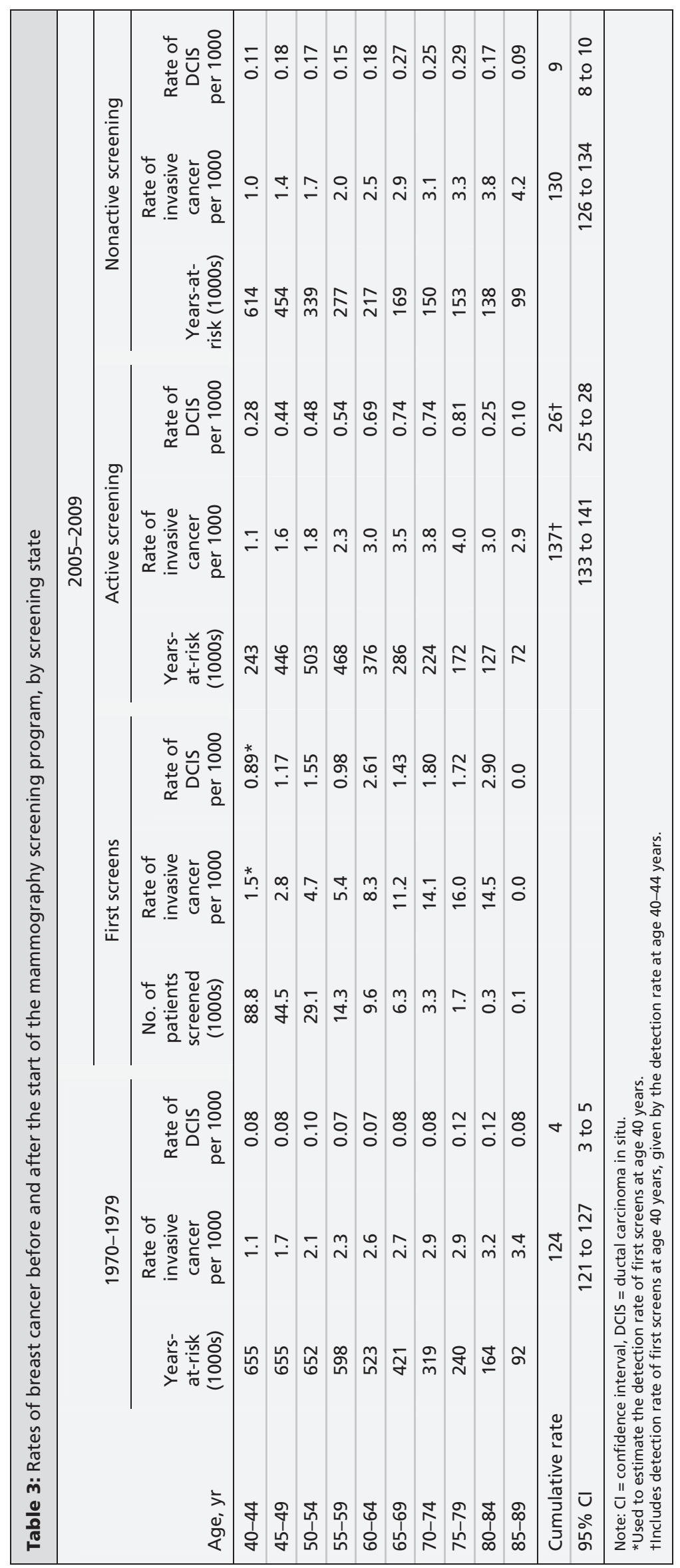


overdiagnosis estimate of about $33 \%$ for invasive and in situ cancer combined. The estimate of overdiagnosis of ductal carcinoma in situ alone was similar to that found in British Columbia. ${ }^{17}$ Adjustment for the effects of time trends, early detection and first screens was limited.

A recent study from Norway $^{18}$ was able to identify women who had undergone screening within the population and allow for a compensatory drop. The authors estimated overdiagnosis rates between $10 \%$ and $20 \%$, depending on the group under consideration.

Thus, the estimate of overdiagnosis in our study is compatible with the estimates seen in randomized trials and more well-controlled population studies.

\section{Limitations}

Several factors should be considered in assessing our study's results. Unlike other studies, we were able to remove the effect of first screens after the age of usual screening initiation. First screens have a high detection rate and can inflate incidence rates. However, the resulting participationbased estimate of overdiagnosis was potentially biased by self-selection for ongoing participation. High socioeconomic status is associated with increased likelihood of screening, ${ }^{19}$ as well as with an increased risk of breast cancer, ${ }^{20}$ which could produce overestimation of the rate of overdiagnosis. The population-based estimate of overdiagnosis is not subject to selection bias and includes allowance for a compensatory drop; however, it involves the projection of cancer rates over 30 years, which is subject to considerable uncertainty. Population estimates of overdiagnosis are likely to be smaller than participation estimates because of dilution by nonparticipants.

\section{Conclusion}

We found a modest association between screening and overdiagnosis of invasive breast cancer after allowing a period of 10 years for a compensatory drop after screening had stopped. Overdiagnosis of invasive disease was confined to screening among women older than 60 years of age. For ductal carcinoma in situ, a period of 10 years may be insufficient, because the lead time may be substantially longer with the likelihood that many identified cases might never lead to invasive cancer. Further research aimed at identifying the time required for the compensatory drop in rates is needed and whether overdiagnosis associated with screening is age dependent. As age increases, both the lead time of screening and the likelihood of competing mortality increase so that the likelihood a woman will survive for the compensatory period declines, and the risk of overdiagnosis increases. It is important to consider the effect of age in discussing screening options with patients and to consider the likelihood of overdiagnosis in the management of ductal carcinoma in situ, especially in older women.

\section{References}

1. Kalager M, Adami HO, Bretthauer M, et al. Overdiagnosis of invasive breast cancer due to mammography screening: results from the Norwegian Screening Program. Ann Intern Med 2012;156:491-9.

2. Morrell S, Barratt A, Irwig L, et al. Estimates of overdiagnosi of invasive breast cancer associated with screening mammography. Cancer Causes Control 2010;21:275-82.

3. Jørgensen KJ, Gotzsche PC. Overdiagnosis in publicly organised mammography screening programmes: systematic review of incidence trends. BMJ 2009;339:b2587.

4. Zahl PH, Gotzsche PC, Maehlen J. Natural history of breast cancers detected in the swedish mammography screening programme: a cohort study. Lancet Oncol 2011;12:1118-24.

5. Elmore JG, Fletcher SW. Overdiagnosis in breast cancer screening: time to tackle an underappreciated harm. Ann Intern Med 2012;156:536-7

6. Elmore JG, Nakano CY, Koepsell TD, et al. International variation in screening mammography interpretations in communitybased programs. J Natl Cancer Inst 2003;95:1384-93.

7. Ravdin PM, Cronin KA, Howlader N, et al. The decrease in breast-cancer incidence in 2003 in the United States. $N$ Engl J Med 2007;356:1670-4.

8. Walter SD, Day N. Estimation of the duration of a pre-clinical disease state using screening data. Am J Epidemiol 1983;118:865-86.

9. Puliti D, Miccinesi G, Paci E. Overdiagnosis in breast cancer: design and methods of estimation in observational studies. Prev Med 2011;53:131-3.

10. Hellquist BN, Duffy SW, Nystrom L, et al. Overdiagnosis in the population-based service screening programme with mammography for women aged 40-49 years in Sweden. J Med Screen 2012;19:14-9.

11. Moss SM, Cuckle H, Evans A, et al. Effect of mammographic screening from age 40 years on breast cancer mortality at 10 years follow-up: a randomised controlled trial. Lancet 2006;368:2053-60.

12. Junod B, Zahl PH, Kaplan RM, et al. An investigation of the apparent breast cancer epidemic in France: screening and incidence trends in birth cohorts. BMC Cancer 2011;11:401.

13. Puliti D, Duffy S, Miccinesi G, et al. Overdiagnosis in mammographic screening for breast cancer in Europe: a literature review. J Med Screen 2012;19(Suppl1):42-56.

14. Biesheuvel C, Barratt A, Howard K, et al. Effects of study methods and biases on estimates of invasive breast cancer overdetection with mammography screening: a systematic review. Lancet Oncol 2007:8:1129-38.

15. de Gelder R, Heijnsdijk EAM, van Ravensteyn NT, et al. Interpreting overdiagnosis estimates in population-based mammography screening. Epidemiol Rev 2011;33:111-21.

16. Duffy SW, Lynge E, Jonsson H, et al. Complexities in the estimation of overdiagnosis in breast cancer. Br J Cancer 2008;99:1176-8.

17. Bleyer A, Welch HG. Effect of three decades of screening mammography on breast-cancer incidence. N Engl J Med 2012;367: 1998-2005.

18. Falk R, Hofvind S, Skaane P, et al. Overdiagnosis among women attending a population-based mammography screening program. Int J Cancer 2013;133:705-12.

19. Canadian Partnership Against Cancer. Breast cancer control in Canada: a system performance special focus report. Toronto (ON): The Partnership; 2012. Available: www.cancerview.ca /systemperformancereport (accessed 2012 Feb. 8).

20. Colditz GA, Baer HJ, Tamimi RM. Breast cancer. In: Schottenfeld $\mathrm{D}$, Fraumeni J, editors. Cancer epidemiology and prevention. 3rd ed. New York (NY): Oxford University Press; 2006. p. 997.

Affiliations: British Columbia Cancer Agency, Vancouver, BC

Contributors: Both authors contributed to the design, analysis and writing of this study. Both authors approved the final version of the manuscript submitted for publication.

Funding: No explicit funding was provided for this study, which was performed by staff of the British Columbia Cancer Agency, Surveillance and Cancer Outcomes.

Acknowledgement: The authors acknowledge the assistance of the reviewers and editor who provided many helpful suggestions. 